\title{
Ordering of the RGB space with a growing self- organizing network. Application to color mathematical morphology
}

\author{
Francisco Flórez-Revuelta \\ U.S.I. “Informática Industrial y Redes de Computadores”. Universidad de Alicante \\ P.O. Box 99. E-03080 Alicante, Spain \\ florez@dtic.ua.es \\ http: //www.ua.es/i2rc
}

\begin{abstract}
Mathematical morphology is broadly used in image processing, but it is mainly restricted to binary or greyscale images. Extension to color images is not straightforward due to the need of application to an ordered space with an infimum and a supremum. In this paper a new approach for the ordering of the RGB space is presented. The adaptation of a linear growing self-organizing network to the three-dimensional color space allows the definition of an order relationship among colors. This adaptation is measured with the topographic product to guarantee a good topology-preservation of the RGB space. Once an order has been established, several examples of application of mathematical morphology operations to color images are presented.
\end{abstract}

\section{Introduction}

Mathematical morphology (MM) has been broadly used in image processing. MM must be applied to a set provided with an order and with a supremum and an infimum pertaining to that order [1]. This is the reason why MM has been mainly applied to binary or grayscale images. Extension to color images is not straightforward, because those two requirements are missing in color spaces, where there is not an order relationship among colors.

Several techniques have been developed to extend MM to color images, getting partial or total orderings of different color spaces (RGB, HSI, YIQ, CIELAB,...). In marginal ordering [2] MM operations are applied to each image channel, recombining the partial results to get the final image. This method is not generally valid because different or new colors can appear due to this separate filtering.

Another strategy is to treat the color at each pixel as a vector. Order is established by reducing each multivariate color to a ranked single value [3], [4], [5], [6]. 


\section{Ordering of the RGB space with a self-organizing network}

Self-organizing neural networks carry out a reduced representation of a vector space in order to achieve some goals [7]: quantization error minimization, entropy maximization, feature mapping, clustering,...

Adaptation of a network of less dimensionality than the input space causes the creation of folders given that the network tries to preserve its topology. In case of a one-dimensional network the final result is similar to a Peano space filling curve [8].

This effect will allow the ordering of the three-dimensional RGB space with a linear self-organizinf network. A modification of the Growing Cell Structure model [9] will be used, setting two neurons at points representing black and white colors in the RGB space.

\subsection{Ordering growing self-organizing network}

In the RGB space, each color is represented by three components, each one of them taking real values in the range $[0,1]$.

The ordering neural network is composed of:

- a set $\mathcal{A}$ of nodes (neurons). Each neuron $c \in \mathcal{A}$ has an associated reference vector $w_{c} \in[0,1]^{3} \subset \mathbb{R}^{3}$. The reference vectors can be regarded as positions in the RGB space of their corresponding neurons,

- a set $C$ of edges (connections) between pairs of neurons. Those connections are not weighted and their purpose is to define the topological structure.

The learning algorithm to approach the network to the RGB space is as follows:

1. Initialize the set $\mathcal{A}$ with three neurons

$$
\mathcal{A}=\left\{c_{0}, c_{1}, c_{2}\right\}
$$

where $c_{0}=(0,0,0), c_{1}=(1,1,1)$ and $c_{2}$ is randomly chosen from the RGB space. Neurons $c_{0}$ y $c_{1}$ will be the extremes of the network, placed in the black and white colors respectively.

2. Connection set $C$ is initialized joining the extremes to neuron $c_{2}$, such that the network is one-dimensional:

$$
C=\left\{\left(c_{0}, c_{2}\right),\left(c_{1}, c_{2}\right)\right\}
$$

3. Generate at random an input signal $\xi \in[0,1]^{3}$ with a uniform distribution.

4. Determine the nearest neuron (winner neuron) $s$ :

$$
s(\xi)=\arg \min _{c \in \mathcal{A}}\left\|\xi-w_{c}\right\|
$$

5. Add the squared distance between the input signal and the winner neuron to an error variable $E_{s}$ :

$$
\Delta E_{s}=\left\|\xi-w_{s}\right\|^{2}
$$


6. Adapt the reference vectors of $s$ and its topological neighbors (neurons connected to $s$ ) towards $\xi$ by a learning step $\mathcal{E}_{s}$ and $\mathcal{E}_{n}$, respectively, of the total distance:

$$
\begin{gathered}
\Delta w_{s}=\mathcal{E}_{s}\left(\xi-w_{s}\right), \quad s \in \mathcal{A}-\left\{c_{0}, c_{1}\right\} \\
\Delta w_{n}=\varepsilon_{n}\left(\xi-w_{n}\right), \quad \forall n \in \mathcal{N}_{s}-\left\{c_{0}, c_{1}\right\}
\end{gathered}
$$

where $\mathcal{N}_{s}$ denotes the set of neighbors of $s$.

7. Every certain number $\lambda$ of input signals generated, insert a new neuron as follows:

- $\quad$ Determine the neuron $q$ with the maximum accumulated error:

$$
q=\arg \max _{c \in \mathcal{A}} \mathcal{E}_{c}
$$

- Insert a new neuron $r$ between $q$ and its further neighbor $f$ :

$$
w_{r}=0.5\left(w_{q}+w_{f}\right)
$$

- Insert new edges connecting the neuron $r$ with neurons $q$ and $f$, removing the old edge between $q$ and $f$.

- $\quad$ Decrease the error variables of neurons $q$ and $f$ multiplying them with a constant $\alpha$. Set the error variable of $r$ with the mean value of $q$ and $f$.

8. Decrease all error variables by multiplying then with a constant $\beta$.

9. If the net size is not yet achieved, go to step 3 .

Figure 1 shows the result of the adaptation of different networks to the RGB space and the sequence of reference vectors from black to white color.

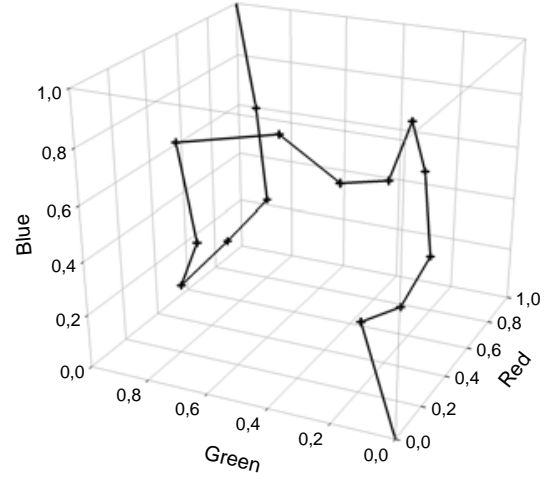

(a)

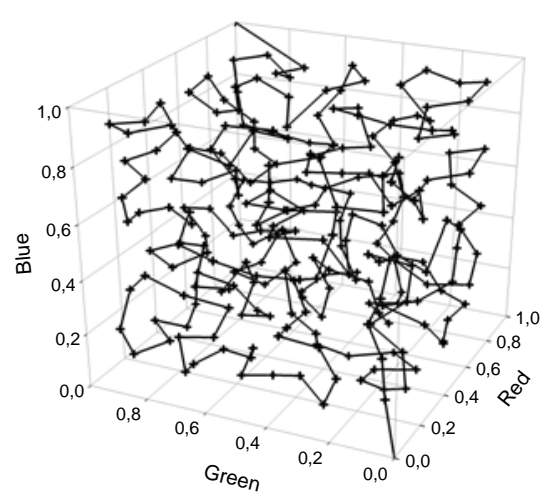

(b)

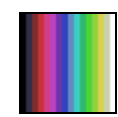

(c)

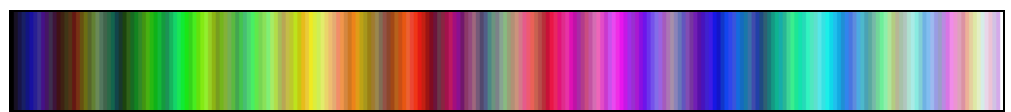

(d)

Fig. 1. Adaptation of a growing self-organizing network to the RGB space: (a) 16 and (b) 256 neurons; and their corresponding color orderings: (c) 16 and (d) 256 reference vectors 


\subsection{Color order}

A mapping of the RGB space onto the neural network is obtained once the learning process is finished :

$$
\phi:[0,1]^{3} \rightarrow \mathbb{N}, \xi \in[0,1]^{3} \rightarrow \phi(\xi) \in \mathbb{N}
$$

where $\phi(\xi)$ is obtained from:

$$
\left\|\mathrm{w}_{\phi(\xi)}-\xi\right\|=\min _{\mathrm{i} / \mathrm{c}_{\mathrm{i}} \in \mathrm{A}}\left\|\xi-\mathrm{w}_{\mathrm{c}}\right\|
$$

However, two different colors can be mapped to the same neuron. This fact establishes a preorder relationship, not being able to ensure the uniqueness of infimum or supremum in a mathematical morphology operation.

In order to avoid this problem, color order is defined as:

$$
\xi_{1}<\xi_{2} \quad \text { if }\left\{\begin{array}{c}
i<j / c_{i}=\phi\left(\xi_{1}\right), c_{j}=\phi\left(\xi_{2}\right) \\
c=\phi\left(\xi_{1}\right)=\phi\left(\xi_{2}\right) \wedge\left\|\xi_{1}-w_{c}\right\|<\left\|\xi_{2}-w_{c}\right\|
\end{array}\right.
$$

Even so, two colors could be at the same distance from the reference one. To solve this problem, one possible criterion is choosing the first point found in the calculation of the infimum or the supremum [10] (figure 2).

\begin{tabular}{|c|c|c|}
\hline 1 & 2 & 3 \\
\hline 4 & 5 & 6 \\
\hline 7 & & \\
\hline & 8 & 9 \\
\hline
\end{tabular}

Fig. 2. Calculation route with a structuring element of size $3 \times 3$

\section{Application to color mathematical morphology}

Once a color order $\phi$ has been defined, thereby allowing the choice of a supremum and infimum from a set of vectors; one can define the basic morphological operators.

The erosion of a digital image $\mathrm{I}: \mathbb{N}^{2} \rightarrow \mathbb{R}^{3}$ at point $(\mathrm{x}, \mathrm{y})$ by structuring element $\mathrm{B}$ is

$$
\varepsilon_{\mathbb{B}}^{I(x, y)}=\inf _{(s, t) \in \mathbb{B}} \phi[I(x+s, y+t)-6(s, t)]
$$

and the corresponding dilation is

$$
\delta_{\mathcal{B}}^{I(x, y)}=\sup _{(s, t) \in \mathbb{B}} \phi[I(x-s, y-t)+6(s, t)]
$$

Usually, when been applied to images, $\mathrm{b}(\mathrm{s}, \mathrm{t})=0, \forall(\mathrm{s}, \mathrm{t}) \in \mathrm{B}$, getting the socalled function-and-set-processing (FSP) filters: 


$$
\begin{aligned}
& \mathcal{E}_{\mathcal{B}}^{I(x, y)}=\inf _{(s, t) \in \mathcal{B}} \phi[I(x+s, y+t)] \\
& \delta_{\mathbb{B}}^{I(x, y)}=\sup _{(s, t) \in \mathcal{B}} \phi[I(x-s, y-t)]
\end{aligned}
$$

From these elementary operations there can be defined a wide set of morphologic operations as opening, closing, gradients or top-hats.

\section{Results and experimentation}

One million networks have been adapted, taking the following learning parameters: size $=256$ neurons, $\varepsilon_{1}=0.1, \varepsilon_{2}=0.01, \lambda=10000, \alpha=0, \beta=0$. Quality of the adaptation can change due to the random behaviour of the learning process. So, topologypreservation has been calculated with the topographic product [11].

The best result has been employed for obtaining the reduced ordering of the RGB space. The examples in this section use an image of the painting "Le chanteur" by Joan Miró and a square structuring element of size 5x5. Figure 3 shows the results of applying different color morphological operations.

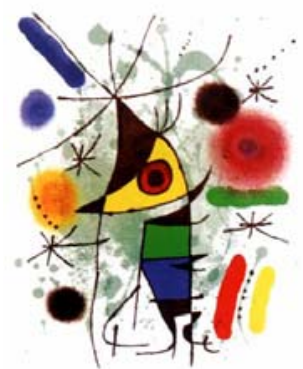

(a)

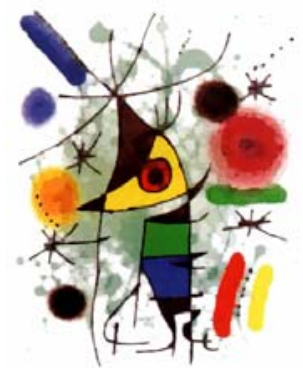

(d)

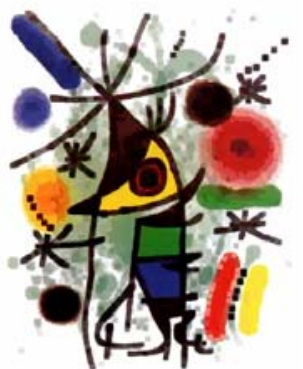

(b)

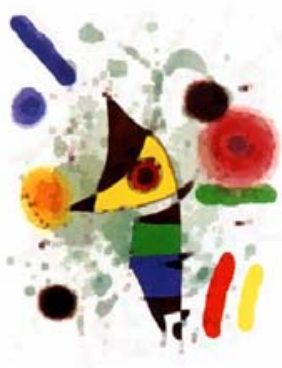

(e)

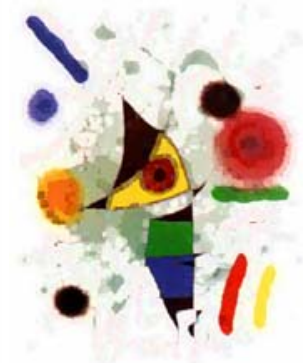

(c)

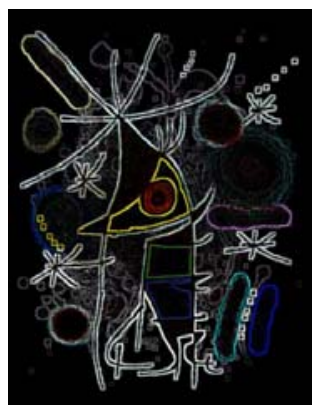

(f)

Fig. 3. (a) Original image, (b) erosion, (c) dilation, (d) opening, (e) closing and (f) gradient by erosion 


\section{Conclusions and current works}

In this paper a new method for the ordering of the RGB space is presented, mainly directed to its later application to mathematical morphology. This ordering is better, in sense of topology-preservation, than other methods; and can be easily implemented in a parallel hardware architecture in order to ensure a fast image processing.

Nowadays, works are focused on the extension of this approach to other color spaces as HIS or YIQ; studying the right learning parameters to be used in each case. Next, ordering will be carried out to other image feature spaces, for instance, to textures.

Outcoming orderings will be applied to different image processing applications as hand gesture recognition, robotics and automatic visual inspection, in which my research laboratory is involved.

\section{Acknowledgements}

This work has been partially funded by the Spanish Ministry of Science and Technology under project DPI2002-04434-C04-01.

\section{References}

1. Serra, J.: Image Analysis and Mathematical Morphology Volume 2: Theoretical Advances. London: Academic Press (1988)

2. Barnett, V.: The ordering of multivariate data. Journal of the Royal Society A, vol 139 (1976) 318-355

3. Comer, M.L., Delp, E.J.: Morphological Operations for Color Image Processing. Journal of Electronic Imaging, Vol. 8, No. 3 (1999) 279-289

4. Chanussot, J., Lambert, P.: Total ordering based on space filling curves for multivalued morphology. In Proceedings of the International Symposium on Mathematical Morphology (ISMM'98) (1998) 51-58

5. Peters II, R.A.: Mathematical morphology for angle-valued images. In Non-Linear Image Processing VIII. SPIE volume 3026 (1997)

6. Hanbury, A., Serra, J.: Mathematical Morphology in the HLS Colour Space. In Proceedings of the $12^{\text {th }}$ British Machine Vision Conference (2001) 451-460

7. Fritzke, B.: Some Competitive Learning Methods. Draft Paper (1997)

8. Kohonen, T.: Self-organizing maps. Springer (1995)

9. Fritzke, B.: Growing Cell Structures - A Self-organizing Network for Unsupervised and Supervised Learning. Neural Networks, Vol. 7, No. 9 (1994) 1441-1460

10. Ortiz, F.G.: Procesamiento morfológico de imágenes en color. Aplicación a la reconstrucción geodésica. Ph. D. Thesis, Universidad de Alicante (2002)

11. Bauer, H.-U., Pawelzik, K.R.: Quantifying the Neighborhood Preservation of SelfOrganizing Feature Maps. IEEE Transactions on Neural Networks, 3(4) (1992) 570-578 